\title{
Automated Cyberbullying Detection and Prevention System
}

\author{
Dr. Egho-Promise Ehigiator Iyobor \\ Regional Technical Head \\ Glo Mobile Ghana Limited \\ Tamale, Ghana \\ Janet Acquah \\ Department of Computer Science \\ Faculty of Applied Science and Technology \\ Koforidua Technical University, Ghana
}

\author{
Richard Antwi Birikorang \\ Department of Computer Science \\ Faculty of Applied Science and Technology \\ Koforidua Technical University, Ghana \\ Jephter Kankam \\ Department of Computer Science \\ Faculty of Applied Science and Technology \\ Koforidua Technical University, Ghana
}

\author{
Emmanuel Akomeah Arhin \\ Department of Computer Science \\ Faculty of Applied Science and Technology \\ Koforidua Technical University, Ghana
}

\begin{abstract}
Every social media platform involves exchange of information between two or more persons. The aim is to perform some online activities. These activities could be buying of gods and services or exchange of false or harmful messages from one person to another. This type of harmful messages could be detrimental to the receiver's reputation.
\end{abstract}

Cyberbullying is the activity performed in cyberspace which involve exchange of intimidation, depression or any negative messages from one person to another. This type of bullying has constituted a big challenge to the youngsters.

Cyberbullying will continue to cause more harm to people on the cyberspace if some measures are not put in place to detect and prevent it. In this study, a system will be designed and developed to detect and prevent harmful messages being sent on the cyberspace.

Keywords:- Cyberbullying, Cyberspace.

\section{INTRODUCTION}

Cyberbullying involves the use of electronic devices such as mobile phones or computers to send embarrassing or defamatory messages over network. According to [1], cyberbullying is a new method of bullying with diverse differences in form, personality, and response.

This is frequent in social media platforms such as Facebook, WhatsApp, Twitter, Instagram, etc. cyberbullying can cause depression, humiliation, anger, isolation and illness.

Besides social media where cyberbullying can be committed, other platforms include messages sent via mobile, online chatting on the internet, emails and message boards such reddit, to mention but a few.
In this research, a system will be designed and developed to detect and prevent cyberbullying

\section{Problem statement}

Cyberbullying has a lot of adverse effects on the target person. It has caused so much depression, humiliation, anger and emotional pain to the victim.

\section{Significance of the problem}

The wellbeing and lifespan of individuals subjected to cyberbullying will be improved with the help of the system since it can detect and prevent cyberbullying.

\section{Objective}

The objective of the research is to detect and prevent cyberbullying

\section{LITERATURE REVIEW}

Cyberbullying is the use of any electronics devices such as mobile phones or computers to send harmful messages to someone.

According to [2], they discovered the use of multi modal data removed from Instagram social media to detect cyberbullying.

In the view of [3], cyberbullying was detected in Twitter and Instagram social media by taking integrating bully and victims scores as the major characteristics.

All the above literatures reviewed could only detect cyberbullying but there was non to prevent it. In this study, automated system will be designed and developed not only to detect cyberbullying but also to prevent it. 
Types of cyberbullying

Cyberbullying can be categorized into different types namely:

- Harassment

- Exclusion

- Outing/doxing

- Trickery

- cyberstalking

- Fraping, masquerading

- Dissing

- Trolling

- Flaming

Benefit of the automated cyberbullying detection and prevention system include:

- It protects the safety and wellbeing of individuals who carryout activities online

- It reduces the rate of cyberbullying on the internet

- It prevents defamatory or malicious messages from getting to the target person

\section{METHODOLOGY}

Qualitative research approach was adopted in this research which is basically an open-ended approach that does not involve hypothesis but gives in depth insight into problems [4]. Qualitative research method was implemented specifically interview.

\section{Data collection instrument}

Interview was used to collect data and this type of instrument was chosen to gather data because is a conversation base research method.

\section{Agile Model}

Agile is a very common software development life cycle model and it will be used in developing the proposed system because of its speedy and elastic response to changes. model.

The figure 1 below shows the different phases in agile

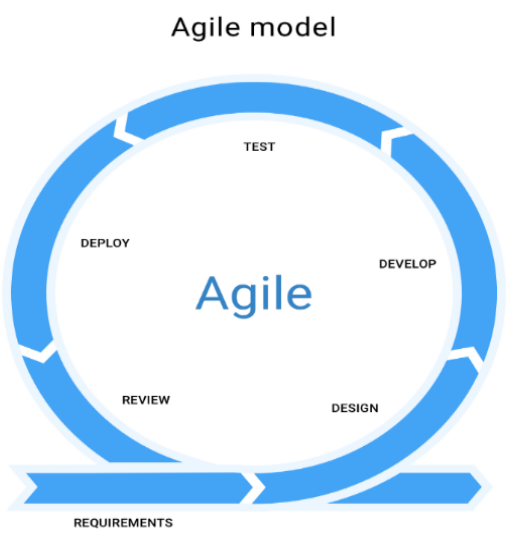

Fig 1:- Represent the phases of Agile Model

\section{Benefits of Agile Model}

- There is efficiency in software development.

- It allows new requirements to be implement easily while software development is in progress.

> Hardware requirements

- Processor: Intel Celeron

- RAM: 2 GB or more

- Hard Disk: 200GB or more

- Operating system: Windows/macOS/Linux

\section{Software requirements}

- Front End: HTML5, CSS3, Bootstrap and Typescript

- Back End: PHP, Laravel, MYSQL

Database Design


Fig 2:- This represents the proposed system database design

\section{Software Design Tool}

The below figure shows a flowchart of the proposed system. It displayed the logical flow and graphical representation of the proposed automated cyberbullying prevention and detection system.

The Figure 3: The figure below shows the logical flow and graphical representation of the proposed system.

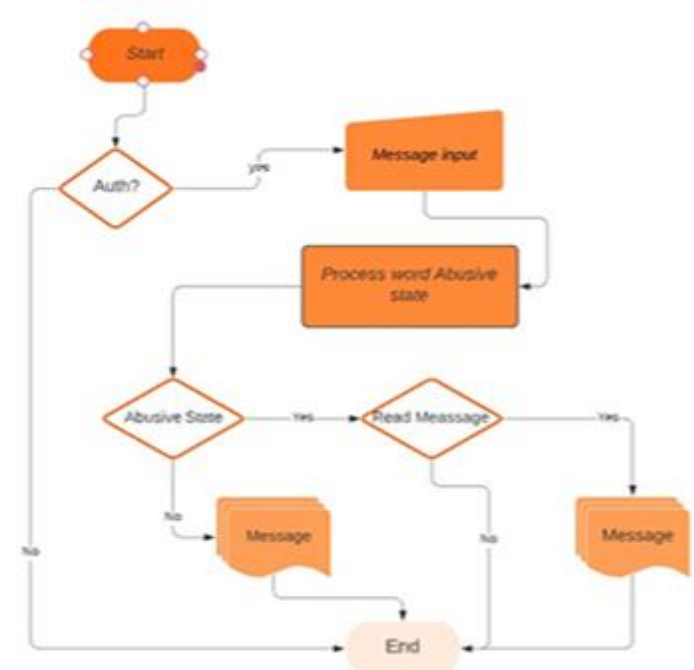

Fig 3:- Flowchart diagram 


\section{EXPERIMENTAL RESULTS AND DATA ANALYSIS}

This session describes how the system was developed and tested.

\section{Programming Tools}

The following programming tools were used to develop the automated cyberbullying detection and prevention system namely:

- Microsoft windows 10 operating system

- Cascading style sheet

- HTML

- Laravel

- PHP

\section{Testing}

Different tests were conducted to determine the system's operational, transitional and maintenance characteristic usability. All the tests executed showed that the system is reliable, efficient, reusable, portable, adaptable, maintainable and scalable.

\section{Usability Test}

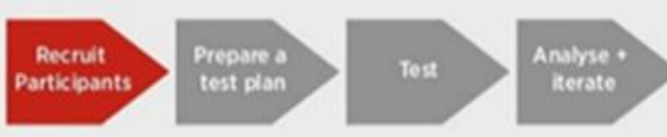

Fig 4:- Usability test. It showed how usability test was done

\section{System Testing}

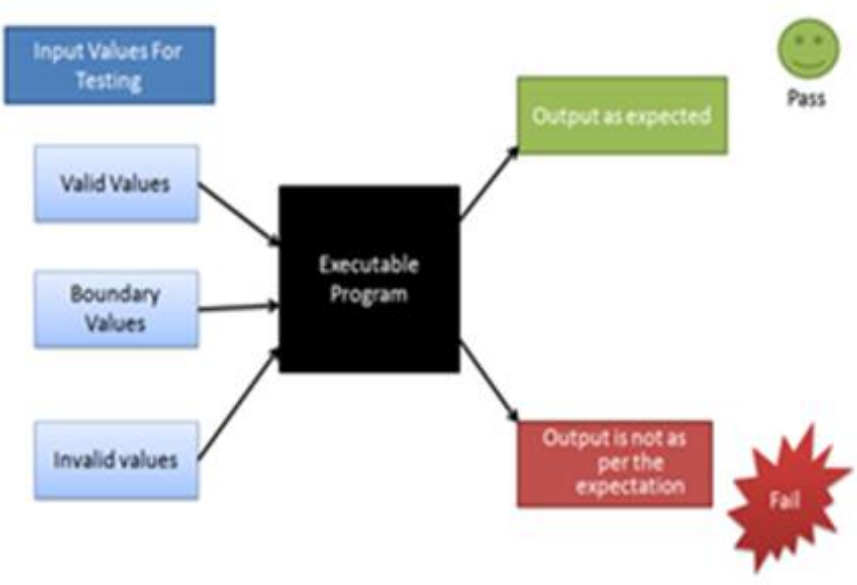

Fig 5:- It demonstrates how the system was tested

\section{Code Testing}

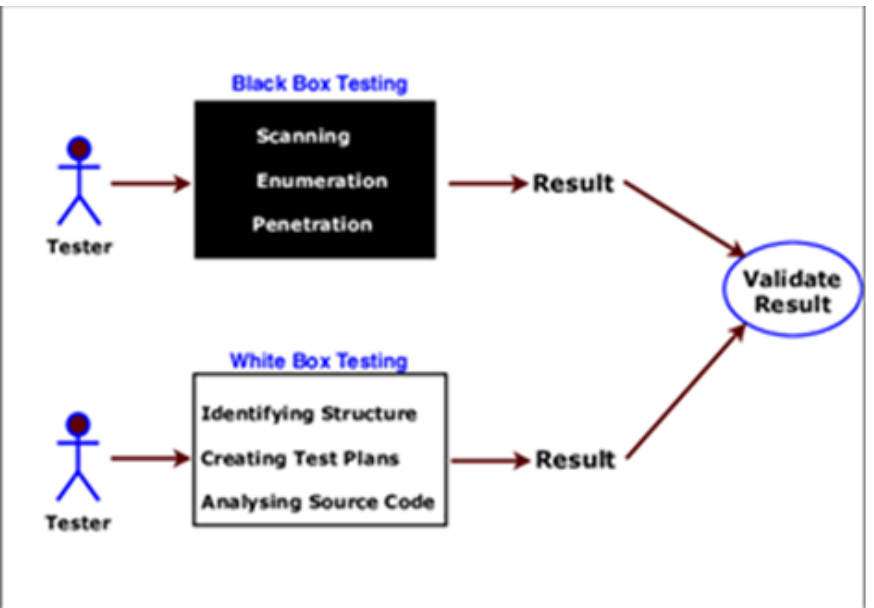

Fig 6:- It showed how black box and white back codes testing were executed.

Detection and Prevention cyberbullying messages



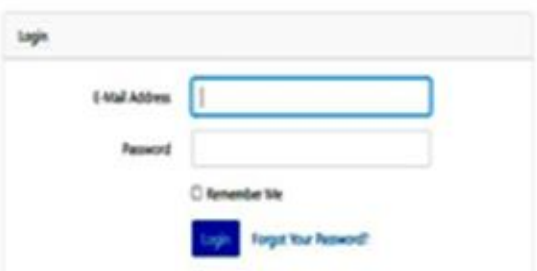

Fig 7:- It is the interface where incoming messages are filtered. Any cyberbullying message that is detected is immediately discarded.

\section{Cyberbullying Message}

$$
\text { Cpe Bling censon som }
$$

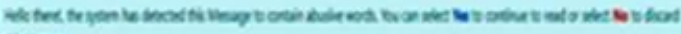$$
\text { novap }
$$

Fig 8:- The above figure showed that cyberbullying message was detected and it was instantaneously deleted. 


\section{CONCLUSION}

The experimental results and data analyses above showed that we have successfully designed and developed automated system to detect and prevent cyberbullying and help to improve the wellbeing and lifespan of individuals who perform online transactions or other activities on any electronics devices which can process and store data.

\section{REFERENCES}

[1]. Qing,L.(2010, May). Cyberbullying in High Schools: A Study of Students' Behaviors and Beliefs about This New Phenomenon. Journal of Aggression, Maltreatment \& Trauma. DOI: 10.1080/10926771003788979

Hosseinmardi, H., Arredondo, S. M., Rahat, I. R., Han, R. (2016) Detection of cyberbullying incidents on the Instagram social network. ResearchGate. Retrieved from https://www.researchgate.net/publication/273640275_ Detection_of_Cyberbullying_Incidents_on_the_Instag ram_Social_Network

[2]. Raisi, E., Huang, N. (2017). Cyberbullying Detection with Weakly Supervised Machine Learning. Retrieved from http://people.cs.vt.edu/ bhuang/papers/raisiasonam17.pdf

[3]. Bhandari, P. (2020, July 30). Methodology. Scribbor. Retrieved from https://www.scribbr.com/methodology/qualitativeresearch/

[4]. Nandhini, B. S. (2015). Online Social Network Bullying Detection Using Intelligence Techniques: Procedia Computer Science. Retrieved from https://www.sciencedirect.com/science/article/pii/S18 7705091500321X?via\%3Dihub 